\title{
EFFICIENT ESTIMATOR OF PROBABILITIES OF LARGE POWER SPILLS IN AN STAND-ALONE SYSTEM WITH WIND GENERATION AND STORAGE
}

\author{
Debarati Bhaumik, Daan Crommelin and Bert Zwart \\ CWI Amsterdam \\ Science Park 123 \\ Amsterdam,1098 XG, NETHERLANDS
}

\begin{abstract}
The challenges of integrating unpredictable wind energy into a power system can be alleviated using energy storage devices. We assessed a single domestic energy system with a wind turbine and a battery. We investigated the best operation mode of the battery such that the occurrence of large power spills is minimized. To estimate the small probability of large power spills, we used the splitting technique for rare-event simulations and to do so, we formulated an appropriate Importance Function such that the workload of the probability estimator is reduced compared to the conventional Crude Monte Carlo estimator. We find that the ramp constraint imposed on the charging/discharging rate of the storage device plays a major role in minimizing large power spills. A new charging strategy for the battery is applied to reduce the large power spills further which results in a trade-off between reductions in large and average power spills, respectively.
\end{abstract}

\section{INTRODUCTION}

To enhance the efficiency of renewable energy generation, it is important to store the generated excess power in the system. We asses a stand-alone single domestic power system with local wind generation and demand supplemented with an energy storage device (a battery) and find the best way to operate the battery such that the probability of large power spills (PLPS) is minimal.

We formulate models for simulating the wind speeds and power demand such that the invariant probability densities of the model data are comparable to the data from measurements. Using these models we analyze how the ramp constraints, the imposed maximal charging/discharging rates on the battery affect the PLPS. We define a new charging strategy for the battery to reduce the PLPS further but it results in the increase of the average power spill. We study the trade-off between reducing the PLPS and reducing the average power spilled by the system.

The probability of occurrence of large power spills is small, hence using CMC probability estimator becomes computationally intensive. We use a variant of the splitting technique for rare-event simulations called the Fixed Number of Successes (FNS) proposed in (Amrein and Künsch 2011) for calculating the PLPS. For our stochastic power system we formulate an appropriate Importance Function (IF) which plays the most significant role in the efficiency of splitting.

\section{MODEL SET UP}

Let the power mismatch between the wind power generation $W(t)$ and demand $D(t)$ at time $t$ be defined as $P(t):=W(t)-D(t)$. The battery is modeled according to $\frac{d B}{d t}:=\alpha P(t), 0<\alpha \leq 1$ and is subjected

to constraints, i.e., the ramp constraint: $-\gamma \leq \frac{d B}{d t} \leq \beta$, where $\gamma<0<\beta$ and the capacity constraint: $0 \leq B(t) \leq B_{\max }$ for all $t \in[0, T]$. Thus, in principle, the battery is charged when $P(t)>0$ and discharged 
if $P(t)<0$ unless any/both of the battery constraints are met. For simulations we take $\gamma=-\beta, \alpha=1$, $T=24$ hours, $d t=0.01$ hours, $B(0)=B_{\max } / 2$ and $F^{*}=850 \mathrm{~W}$.

Power Spill: Let $F(t):=P(t)-\tilde{P}(t)$ be the residual power in the system, where $\tilde{P}(t)$ is the power getting absorbed or delivered by the battery. When $F(t)>0$, power spill occurs: there is more power production than demand and the battery cannot absorb all the excess power because of the battery constraints. The PLPS over a time length $T$ is given by $\gamma:=\mathscr{P}\left(\sup _{t \in[0, T]}\{F(t)\} \geq F^{*}\right)$, where $F^{*}>0$.

Importance Function: We formulate the IF as the distance of the system from rare event sets in the phase space of $B(t)$ and $P(t)$. There are two rare event sets for this model. Set 1 occurs when the battery is fully charged to $B_{\max }$ and $P(t) \geq F^{*}$ and set 2 occurs when the battery cannot absorb all the power because of the imposed ramp constraints, i.e. when $P(t) \geq F^{*}+\beta$.

New charging Scheme: To reduce the PLPS further a fraction of the battery $1-\varepsilon$ is reserved for absorbing only those values of excess power which are greater than $F^{*}$, where $0 \leq \varepsilon \leq 1$.

\section{RESULTS}

We study how the PLPS varies with $\beta$ for different $B_{\max }$. It is observed that PLPS reduces with $\beta$ till an optimal value $\beta^{*}$ where it is minimal, then again increases and becomes constant. This imply either a very fast or a very slow charging/discharging battery leads to more power spills. The value of PLPS around $\beta^{*}$ reduces with increasing $B_{\max }$.

Implementing the new charging scheme shows that, as the value of $\varepsilon$ reduces, PLPS gets smaller. The more we reserve the battery for only storing values of generated excess power greater than $F^{*}$ the lower the PLPS gets. PLPS drops by a factor of 100 when $\varepsilon$ is reduced from 1 to 0.8 . The reduction in $\varepsilon$ nominally increases the average power spill.

We find that the proposed IF for FNS splitting technique significantly enhances the time efficiency of FNS over CMC. It is observed that for PLPS $\sim 10^{-3}$ FNS is 60 times faster than CMC and for PLPS $\sim 10^{-4}$ FNS is 300 times faster than CMC.

A much more detailed presentation and discussion of these findings including numerical details and results can be found in (Bhaumik, Crommelin, and Zwart 2015)

\section{REFERENCES}

Amrein, M., and H. R. Künsch. 2011. "A variant of importance splitting for rare event estimation: Fixed number of successes". ACM Transactions on Modeling and Computer Simulation (TOMACS) 21 (2): 13.

Bhaumik, Debarati, and Crommelin, Daan and Zwart, Bert. 2015. "Mitigation of Large Power spills by a Storage Device". http://persistent-identifier.org/?identifier=urn:nbn:nl:ui:18-23525. Submitted. 